\title{
Transmission delay times of localized waves
}

\author{
H. Schomerus \\ Instituut-Lorentz, Universiteit Leiden, P.O. Box 9506, 2300 RA Leiden, The Netherlands \\ and Max-Planck-Institut für Physik komplexer Systeme, Nöthnitzer Strasse 38, 01187 Dresden, Germany
}

(Received 5 March 2001; published 18 July 2001)

\begin{abstract}
We investigate the effects of wave localization on the delay time $\tau$ (frequency sensitivity of the scattering phase shift) of a wave transmitted through a disordered waveguide. Localization results in a separation $\tau=\chi$ $+\chi^{\prime}$ of the delay time into two independent but equivalent contributions, associated to the left and right end of the waveguide. For $N=1$ propagating modes, $\chi$ and $\chi^{\prime}$ are identical to half the reflection delay time of each end of the waveguide. In this case the distribution function $P(\tau)$ in an ensemble of random disorder can be obtained analytically. For $N>1$ propagating modes the distribution function can be approximated by a simple heuristic modification of the single-channel problem. We find a strong correlation between channels with long reflection delay times and the dominant transmission channel.
\end{abstract}

DOI: 10.1103/PhysRevE.64.026606

PACS number(s): 42.25.Dd, 42.25.Hz, 72.15.Rn

\section{INTRODUCTION}

In this paper, we characterize localization of randomly scattered waves by means of a dynamical quantity, the delay time $\tau$.

Wave localization is perhaps the most striking effect of multiple random scattering [1-4]_in a waveguide geometry, it results in the exponential attenuation of the transmitted intensity $I(L) \propto \exp (-2 L / \xi)$ for lengths $L$ of the waveguide greater than the localization length $\xi$, even in the absence of absorption. Localization was first investigated in mesoscopic systems [5-7]. Recently the undertaking of its realization and observation for microwaves $[8,9]$ and optical waves [10] has attracted a lot of interest. It is still under debate $[11,12]$ whether some of these observations are due to localization or absorption.

The delay time $\tau=d \phi / d \omega$ is the frequency sensitivity of a scattering phase shift $\phi$, and has been identified by Wigner [13] as a measure of the exploration time of the scattering region (see also Refs. [14,15]). Recent experiments have succeeded in the direct measurement of the so-called singlemode delay time for specified incident and detected modes, both for microwaves [16] and optical waves [17]. (The attribute "single-mode" means here that only one of the $N$ propagating modes is excited, and only one mode is selected for detection, but does not imply any restriction of $N$ itself.) These experimental efforts have promoted the single-mode delay times to quantities of interest in their own right. The measurements have been performed with waveguides shorter than the localization length, and their outcome can be successfully described by diffusion theory [18]. That does not mean that wave localization is of no interest in this contextnote that the experiments on localization and delay times have been performed on the same sorts of sample, by the same groups.

Theoretical work on the localized regime has mostly concentrated on the delay times of the reflected signal [19-26]. Some aspects for the transmission delay-time problem for a single propagating channel $(N=1)$ have been studied in Ref. [27], where it was found that the distribution of $\tau$ has a universal quadratic tail, $P(\tau) \propto \tau^{-2}$, for large $\tau$. This tail eventually crosses over into a log-normal tail, at some large value $\tau_{c}$ that increases with the system length-even though the tail is irrelevant for the direct experimental or numerical investigation of the distribution itself, it is reflected in physical properties of mesoscopic systems (for a review see Ref. [28]). Reference [27] also addressed the properties of a delay-time weighted by the transmission coefficient, which is relevant for the conductance of mesoscopic wires.

In this work we investigate the distribution of the transmission delay time $\tau$ in the localized regime. It will turn out that the transmission and reflection problem are closely related for $N=1$. The transmission delay time is then the mean of the reflection delay times of the both ends of the waveguide, and the exact form of the limiting distribution function $P(\tau)$ for $L \rightarrow \infty$ can be found analytically. At finite length the result is applicable in the range $0<\tau<\tau_{c}$. Because $\tau_{c}$ is very large in the localized regime, this covers the range of delay times that is relevant for direct experimental observation and comparison with numerical simulations.

For $N>1$ there is still only one relevant transmission channel. Consequently, once again localization results in a separation of the transmission delay time into two independent but equivalent contributions from both ends of the waveguide. Moreover, one of the contributions only depends on the excitation mode, while the other only depends on the detection mode. However, the transmission delay times are no longer directly related to the reflection delay times. Nevertheless it is possible to obtain the distribution function of single-mode delay times approximately by a heuristic modification of the single-channel problem.

Although there is no direct relation to the reflection problem for the individual single-mode delay times and $N>1$, there exists an intensity-weighted combination of all delay times that is more closely related to the reflection problem. This combination involves the orthogonal transformation matrix from the basis of transmission channels to the eigenvectors of the Wigner-Smith time-delay matrix. From our numerical simulations we find a strong correlation of the dominant transmission channel and the channel with the largest Wigner-Smith delay time.

The paper is organized as follows. In Sec. II we provide the necessary background material that will be used later on 


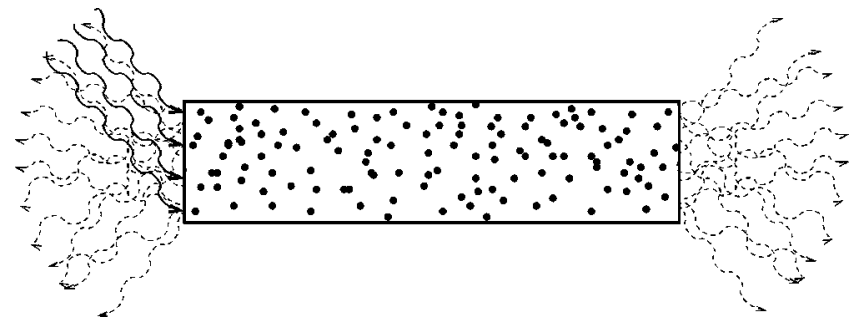

FIG. 1. Quasi-one-dimensional waveguide filled by a disordered medium and illuminated by a monochromatic plane wave. The scattered wave acquires a scattering phase shift $\phi$. We investigate the frequency sensitivity (delay time) $\tau=d \phi / d \omega$ for the transmitted wave.

in the investigation of the transmission delay times. This includes a short review of the diffusive regime and the reflection delay times in the presence of localization. In Sec. III we discuss the case $N=1$ of a single-channel waveguide and calculate the distribution function of the transmission delaytime analytically. Section IV is devoted to waveguides with more than one propagating channel. We will first discuss the single-mode delay times and compare the distribution from a numerical simulation with the analytic expression that arises from the heuristic approximation. Then we turn to the weighted combination of all delay times and use it to investigate the relation of the dominant transmission channel with the channel associated to the largest reflection delay time.

\section{BASIC CONCEPTS}

\section{A. Waveguide geometry}

Figure 1 depicts a quasi-one-dimensional waveguide (length $L$ much larger than the width) that is filled by a medium with randomly placed scatterers (mean free path $l$ ). We assume that there is no absorption and no inelastic scattering inside the waveguide, and consider a monochromatic scalar wave (disregarding polarization) for simplicity. Also we assume that time-reversal symmetry is preserved, as is appropriate for the propagation of light in absence of magneto-optical effects.

The number $N$ of propagating modes at frequency $\omega$ equals the number of transversal excitations inside the waveguide, and is given by $N=\pi \mathcal{A} / \lambda^{2}$ for a waveguide with openings of area $\mathcal{A}$ (here $\lambda=c / \omega$ is the wavelength and $c$ is the propagation velocity of light). In the numerical simulations we will work with a planar waveguide of width $W$ $\ll L$, where $N=2 W / \lambda$. For a unified description we introduce the scattering time $\gamma=\alpha l / c$, with the coefficient $\alpha$ $=2\left(\pi^{2} / 4,8 / 3\right)$ for one-dimensional (two-dimensional, three-dimensional) scattering inside the quasi-onedimensional waveguide, and the relative length $s=\alpha^{\prime} L / l$, with $\alpha^{\prime}=1 / 2(2 / \pi, 3 / 4)$. The localization length is then given by $\xi=(N+1) l / \alpha^{\prime}$.

\section{B. Scattering formalism}

The number $N$ of propagating modes inside the waveguide corresponds to the number of independent incident modes close to each opening of the waveguide. In experi- mental practice these modes can be chosen as plane waves with discretized propagation direction, and mode selection is realized by the choice of the positions of source and detector. In such a single-mode experiment, the waveguide is probed by external illumination with amplitude $\Psi_{m}$ in mode $m$, and the transmitted or reflected signal $\Phi_{n m}$ is detected in mode $n$, with $n, m=1, \ldots, 2 N$. (The modes with index $n, m$ $=1, \ldots, N$ are associated with the left end of the waveguide, while the remaining modes pertain to the right end of the waveguide.) The numbers

$$
S_{n m}=\Phi_{n m} / \Psi_{m}
$$

form the elements of the $2 N \times 2 N$ scattering matrix

$$
S=\left(\begin{array}{cc}
r & t^{\prime} \\
t & r^{\prime}
\end{array}\right)
$$

with four $N \times N$ dimensional blocks that correspond to reflection or transmission with the incident radiation from the left $(r, t)$ or from the right $\left(r^{\prime}, t^{\prime}\right)$. The scattering matrix is unitary due to flux conservation in the absence of absorption, and only depends on one frequency because there are no inelastic processes. Furthermore, the scattering matrix is symmetric due to time-reversal symmetry, hence $t^{\prime}=t^{T}, r$ $=r^{T}$, and $r^{\prime}=r^{\prime T}$.

A useful representation of the scattering matrix is the polar decomposition [7]

$$
S=\left(\begin{array}{cc}
u^{T} & 0 \\
0 & v^{T}
\end{array}\right)\left(\begin{array}{cc}
\sqrt{1-\mathcal{T}} & \sqrt{\mathcal{T}} \\
\sqrt{\mathcal{T}} & -\sqrt{1-\mathcal{T}}
\end{array}\right)\left(\begin{array}{ll}
u & 0 \\
0 & v
\end{array}\right),
$$

with unitary matrices $u$ and $v$ and the diagonal matrix $\mathcal{T}$ $=\operatorname{diag}\left(T_{1}, \ldots, T_{N}\right)$ of transmission eigenvalues (eigenvalues of $\left.t^{\dagger} t\right)$. For convenience we order them by magnitude, $T_{1}>T_{2}>\cdots>T_{N}$.

\section{Intensity and delay time}

The elements of the scattering matrix can be written as

$$
S_{n m}=\sqrt{I_{n m}} \exp \left(i \phi_{n m}\right)
$$

where $I_{n m}$ is the detected intensity for unit incident intensity and $\phi_{n m}$ is the scattering phase shift. The single-mode delay time is defined as the derivative of the scattering phase shift with respect to frequency,

$$
\tau_{n m}=\frac{d \phi_{n m}}{d \omega}=\operatorname{Im} S_{n m}^{-1} \frac{d S_{n m}}{d \omega} .
$$

Its interpretation as an exploration time of the medium stems from the short-wavelength limit. The phase can then be approximated by the classical action $S_{\mathrm{cl}}$ of trajectories (there may be several) that satisfy the boundary conditions of the incident and detected modes. According to classical mechanics, the derivative $d S_{\mathrm{cl}} / d \omega$ of the phase with respect to frequency (energy) equals the classical propagation time through the medium. 


\section{Ballistic case}

In the ballistic regime $s \ll 1$ the wave is transmitted without any attenuation, and the modes can be chosen easily such that each incident mode $m$ is strictly associated with a transmitted mode $n^{\prime}(m)$, namely, by using the reflection symmetry of the waveguide (exchanging left and right). The intensity is then given by $I_{n m}=\delta_{n n^{\prime}}$, and the delay time is $\tau_{n m}$ $=\delta_{n n^{\prime}} L / c_{m}$, where $c_{m}$ is the longitudinal propagation velocity in mode $m$. The average over all modes is $\left\langle L / c_{m}\right\rangle=\gamma s$.

\section{E. Diffusion theory}

Diffusion theory applies when the length $L$ of the waveguide exceeds the mean free path $l$ but is less than the localization length $\xi$. The fluctuations of the intensity $I_{n m}$ for given $m$ and varying $n$ result in a speckle pattern of bright and dark spots, which is described by the Rayleigh distribution

$$
P\left(I_{n m}\right)=\frac{1}{\langle I\rangle} \exp \left(-I_{n m} /\langle I\rangle\right)
$$

The mean intensity per mode is $\langle I\rangle=\langle T\rangle / N$ in transmission and $\langle I\rangle=(1-\langle T\rangle) / N$ in reflection, where [7]

$$
\langle T\rangle=\frac{1}{N}\left\langle\operatorname{tr} t^{\dagger} t\right\rangle=(1+s)^{-1}
$$

is the mean transmission probability. For the special case $n$ $=m$ in reflection the mean intensity doubles due to coherent backscattering [29]. The speckle pattern can also be understood from the uniform distribution of the matrices $u$ and $v$ in the group of unitary matrices $\mathrm{U}(N)$. For large $N$, the elements of $u$ and $v$ can be considered as random Gaussian numbers with variance $\left\langle\left|u_{l m}\right|^{2}\right\rangle=\left\langle\left|v_{l m}\right|^{2}\right\rangle=1 / N$, and the Rayleigh distribution (6) follows from the central-limit theorem. $[16,18]$

The distribution function of the delay time is given by

$$
P\left(\tau_{n m}\right)=\frac{Q}{2\langle\tau\rangle}\left[Q+\left(\tau_{n m} /\langle\tau\rangle-1\right)^{2}\right]^{-3 / 2} .
$$

In transmission $Q=2 / 5$ and $\langle\tau\rangle=\gamma s^{2} / 3$, while in reflection $Q=2 s / 5$ and $\langle\tau\rangle=2 \gamma s / 3$ (for ballistic corrections in reflection, see Ref. [25]).

\section{F. Localized regime}

In the localized regime $L \gtrsim \xi$ the transmission eigenvalues $T_{n}$ become exponentially small, with well-separated, selfaveraging exponents $-\left\langle\ln T_{n}\right\rangle / L=2 n / \xi$. Transmission is dominated by the transmission channel with eigenvalue $T_{1}$, which is exponentially larger than all the other transmission eigenvalues. In terms of the polar decomposition (3),

$$
t_{n m}=\sqrt{T_{1}} v_{1 n} u_{1 m} \Rightarrow I_{n m}=T_{1}\left|v_{1 n} u_{1 m}\right|^{2} .
$$

For large $N$ the complex numbers $v_{1 n}$ and $u_{1 m}$ again can be considered as Gaussian random numbers. For fixed incident mode $m$ and within a given disorder realization (fixed $T_{1}$ ), this results again in the Rayleigh distribution (6) for $I_{n m}$, with $\langle I\rangle=T_{1}\left|v_{1 m}\right|^{2}$. If one also averages over the incident mode, however, one finds

$$
P\left(I_{n m}\right)=\frac{2 N^{2}}{T_{1}} K_{0}\left(2 N \sqrt{I_{n m} / T_{1}}\right),
$$

with $K_{0}$ a modified Bessel function of the second kind. This deviates from the Rayleigh law, obviously because the central limit theorem no longer holds due to the large relative differences between the transmission eigenvalues. The reflected intensities $I_{n m}$, however, still follow the Rayleigh distribution with $\langle I\rangle=1 / N$, since they are governed by the nonfluctuating reflection eigenvalues $R_{i}=1-T_{i} \approx 1$.

Because transmission becomes negligible, the reflection matrices $r=u^{T} u$ and $r^{\prime}=-v^{T} v$ become unitary. The singlemode delay times of reflection can then be related to the Wigner-Smith delay times $\tilde{\tau}_{i}, \tilde{\tau}_{i}^{\prime}$, which are the eigenvalues of the Wigner-Smith matrices

$$
\begin{gathered}
q=-i r^{\dagger} \frac{d r}{d \omega}=u^{\dagger}\left(2 \operatorname{Im} u^{*} \frac{d u^{T}}{d \omega}\right) u, \\
q^{\prime}=-i r^{\prime \dagger} \frac{d r^{\prime}}{d \omega}=v^{\dagger}\left(2 \operatorname{Im} v^{*} \frac{d v^{T}}{d \omega}\right) v,
\end{gathered}
$$

respectively (for details of the relation refer to Refs. [24,25]).

The two sets of Wigner-Smith delay times are independent and equivalent. In terms of the rates $\mu_{i}=\tilde{\tau}_{i}^{-1}$, the joint distribution function is given by the Laguerre ensemble [23]

$$
P\left(\left\{\mu_{i}\right\}\right) \propto \prod_{i<j}\left|\mu_{i}-\mu_{j}\right| \prod_{i} \Theta\left(\mu_{i}\right) e^{-\gamma(N+1) \mu_{i}},
$$

where the step function $\Theta(x)=0$ for $x<0$ and $\Theta(x)=1$ for $x>1$. Equation (12) generalizes earlier results for $N=1$ [1922] to arbitrary $N$.

We order the delay times by their magnitude, $\tilde{\tau}_{1}>\tilde{\tau}_{2}$ $>\ldots>\tilde{\tau}_{N}$. Of special interest is the largest delay time $\tilde{\tau}_{1}$, which is known to dominate the statistics of the reflection delay times $[24,25]$, although to a lesser extent than $T_{1}$ determines the transmitted intensity. Its distribution follows from a result by Edelman [30] for the smallest $\mu$ in the Laguerre ensemble and is given by

$$
P\left(\tilde{\tau}_{1}\right)=\frac{\gamma N(N+1)}{\widetilde{\tau}_{1}^{2}} \exp \left[-\gamma N(N+1) / \widetilde{\tau}_{1}\right]
$$

The mean $\left\langle\tilde{\tau}_{1}\right\rangle$ diverges because of the quadratic tail for large $\tilde{\tau}_{1}$. These large fluctuations are a signature of localization $[26,27,31,32]$, and have been interpreted as exploration of the localized regions deep inside the waveguide. Our result for the transmission delay time will support this interpretation: We will see in Sec. IV D that the corresponding eigenvector of the Wigner-Smith matrix is correlated with the dominant transmission channel. 


\section{SINGLE-CHANNEL WAVEGUIDE}

The distribution of the transmission delay time $\tau_{12}$ for a single propagating mode $(N=1)$ has been investigated previously in Ref. [27], where it was found that $P\left(\tau_{12}\right) \propto \tau_{12}^{-2}$ for large $\tau_{12}$. In this Section we will calculate the distribution function analytically, for all $\tau_{12}$.

For $N=1$, the scattering matrix is a $2 \times 2$ matrix, hence the transmission and reflection elements $t=u v \sqrt{T}, r$ $=u^{2} \sqrt{1-T}$, and $r^{\prime}=-v^{2} \sqrt{1-T}$ reduce to complex numbers, while the matrices $u, v$, of the polar decomposition are now unimodular complex numbers. The single-channel case is special because the transmission delay time

$$
\tau_{12}=\operatorname{Im} u^{-1} \frac{d u}{d \omega}+\operatorname{Im} v^{-1} \frac{d v}{d \omega}=\frac{\tau_{11}+\tau_{22}}{2}
$$

is directly related to the reflection delay times

$$
\begin{gathered}
\tau_{11}=\operatorname{Im} r^{-1} \frac{d r}{d \omega}=2 \operatorname{Im} u^{-1} \frac{d u}{d \omega} \\
\tau_{22}=\operatorname{Im} r^{\prime-1} \frac{d r^{\prime}}{d \omega}=2 \operatorname{Im} v^{-1} \frac{d v}{d \omega} .
\end{gathered}
$$

The relation holds for all lengths (it does not require localization), and can also be derived from the condition of unitarity of the scattering matrix,

$$
r t^{*}+t r^{*}=0 \Rightarrow \frac{d}{d \omega}\left(r t^{*}+t r^{\prime *}\right)=0
$$

It is convenient, also in view of the case $N>1$ to be discussed in Sec. IV, to introduce the quantities

$$
\chi=\operatorname{Im} u^{-1} \frac{d u}{d \omega}, \quad \chi^{\prime}=\operatorname{Im} v^{-1} \frac{d v}{d \omega} .
$$

In the localized regime, the reflection delay times are determined by scattering in nonoverlapping regions close to each end of the waveguide. Hence $\chi$ and $\chi^{\prime}$ become independent, and their joint distribution function $P\left(\chi, \chi^{\prime}\right)=P(\chi) P\left(\chi^{\prime}\right)$ factorizes. The reflection delay times $\tau_{11}=2 \chi, \tau_{22}=2 \chi^{\prime}$ equal the Wigner-Smith delay times $\tilde{\tau}_{1}, \tilde{\tau}_{1}^{\prime}$, respectively. The function

$$
P(\chi)=\frac{\gamma}{\chi^{2}} \exp (-\gamma / \chi) \Theta(\chi)
$$

[and equivalently $P\left(\chi^{\prime}\right)$ ] hence follows from the Laguerre ensemble, Eq. (12), for $N=1$. The derivation in the framework of one-dimensional scaling theory is briefly recapitulated in Appendix A. $P(\chi)$ eventually is determined by the requirement that it becomes independent of length in the localized regime, which results in the stationarity condition

$$
\gamma c \frac{\partial P}{\partial L}=\frac{\partial}{\partial \chi}\left(-\gamma+\frac{\partial}{\partial \chi} \chi^{2}\right) P=0 .
$$

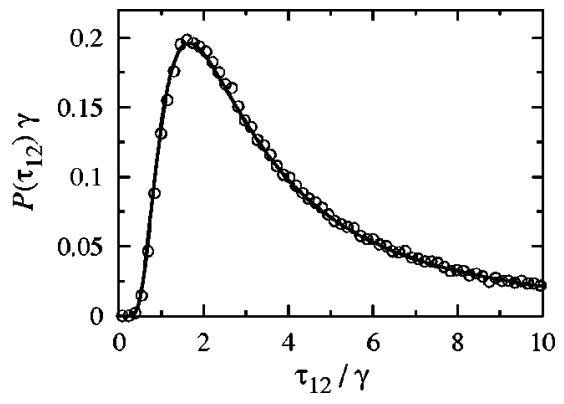

FIG. 2. Distribution of transmission delay time $\tau$ for a singlechannel waveguide. The analytic result (21) (curve) is compared with the results of a numerical simulation of random scattering in a single-channel waveguide.

In Sec. IV B we will propose a slightly modified version of this equation for the case $N>1$.

From Eq. (19), the distribution of the transmission delay time $\tau_{12}=\chi+\chi^{\prime}$ is then found by integration,

$$
\begin{aligned}
P\left(\tau_{12}\right) & =\int_{0}^{\tau_{12}} d x \frac{\gamma^{2}}{x^{2}\left(\tau_{12}-x\right)^{2}} \exp \left[-\gamma / x-\gamma /\left(\tau_{12}-x\right)\right] \\
& =4 \frac{\gamma^{2}}{\tau_{12}^{3}} \exp \left(-\frac{2 \gamma}{\tau_{12}}\right)\left[K_{0}\left(\frac{2 \gamma}{\tau_{12}}\right)+K_{1}\left(\frac{2 \gamma}{\tau_{12}}\right)\right]
\end{aligned}
$$

In Fig. 2 this prediction is compared with the result of a numerical simulation of random scattering in a planar singlechannel waveguide. In these simulations the Helmholtz equation is solved on a square lattice. In terms of the lattice constant $a$, the width of the waveguide is $W=3 a$, and the wavelength is $\lambda=4 a$, giving rise to a single propagating mode. Disorder is modeled by a random on-site potential, with localization length $\xi=4 l=54 a$. The scattering rate $\gamma$ is determined from the ballistic regime. We find perfect agreement between Eq. (21) and the numerical simulations, without any free parameter.

\section{MULTICHANNEL WAVEGUIDE}

Now we turn to the case $N>1$ of more than one propagating mode in the waveguide. We first show that the delay times separate into two independent contributions and discuss some consequences. Then we turn to the distribution function $P\left(\tau_{n m}\right)$ and propose an approximation, based on a heuristic modification of the case $N=1$, which agrees well with the result of numerical simulations. Finally, we investigate the correlation between the transmission channel with eigenvalue $T_{1}$ and the eigenvector of the Wigner-Smith matrix with eigenvalue $\tilde{\tau}_{1}$.

\section{A. Separation rule}

For the transmitted intensity it is sufficient to consider the reduced form $t_{n m}=v_{1 n} u_{1 m} \sqrt{T_{1}}$, Eq. (9), of the transmissionmatrix elements in the localized regime. Under the additional assumption (which is validated by the numerical simulations) that the coefficients $v_{k n}, u_{k m}, k \neq 1$, do not depend much 
more sensitively (by large factors $\propto e^{k L / \xi}$ ) on frequency than the elements $v_{1 n}$ and $u_{1 m}$, this form can also be used for the delay times, which then separate into two contributions,

$$
\begin{gathered}
\tau_{n m}=\chi_{m}+\chi_{n}^{\prime}, \\
\chi_{m}=\operatorname{Im} \frac{1}{u_{1 m}} \frac{d u_{1 m}}{d \omega}, \quad \chi_{n}^{\prime}=\operatorname{Im} \frac{1}{v_{1 n}} \frac{d v_{1 n}}{d \omega} .
\end{gathered}
$$

The contribution $\chi_{m}$ only depends on the mode index $m$ of the incident mode, while $\chi_{n}^{\prime}$ only depends on the detected mode $n$. This gives rise to strong correlations between the delay times for each disorder realization: They obey the relations

$$
\tau_{i j}+\tau_{k l}=\tau_{i l}+\tau_{k j} .
$$

The dependence on the mode indices suggests that $\chi$ and $\chi^{\prime}$ are independent and equivalent, and that they are determined by scattering within a couple of localization lengths close to the associated opening. This is also suggested by the fact that $\chi_{m}$ only depends on the matrix $u$, while $\chi_{n}^{\prime}$ only depends on the matrix $v$. These matrices, on the other hand, determine the reflection matrices $r=u^{T} \sqrt{1-\mathcal{T}} u \approx u^{T} u$ and $r^{\prime} \approx-v^{T} v$, which can be considered as independent in the localized regime. (The approximation $\mathcal{T}=0$ corresponds to neglecting the influence of the opposite end of the waveguide, which is far away). However, that might be deceptive - note that although $u$ and $v$ give $r$ and $r^{\prime}$, they are themselves not uniquely determined by $r$ and $r^{\prime}$ in this approximation: e.g., the same reflection matrix $r$ can be obtained from $o u$, with $o$ an arbitrary orthogonal matrix. The matrix $u$ can only be determined uniquely from $r$ if we also use the information in $t$, which depends on the opposite end of the waveguide. We will demonstrate now that $\chi$ and $\chi^{\prime}$, nevertheless, become independent in the localized regime. However, in Sec. IV C we will see how degrees of freedom which are similar in nature as $o$ reflect in the statistical distribution of the delay times.

In order to demonstrate that $\chi$ and $\chi^{\prime}$ are indeed independent, we cut the waveguide into two parts (associated with subscripts $i=1,2$ ), still requiring that the lengths $L_{i} \gg \xi$. The well-known composition rule

$$
t=t_{2}\left(1-r_{1}^{\prime} r_{2}\right)^{-1} t_{1}
$$

and the relations $1 \gg T_{1, i} \gg T_{k \neq 1, i}$ yield

$$
\begin{gathered}
t_{n m}=v_{1 n, 2} u_{1 m, 1} \sqrt{T_{1}}, \\
T_{1}=T_{1,1} T_{1,2}\left(\left[\left(u_{2}^{*} v_{1}^{\dagger}+u_{2} v_{1}^{\mathrm{T}}\right)^{-1}\right]_{11}\right)^{2} .
\end{gathered}
$$

Note that $T_{1}$ is indeed real. This gives $\tau_{n m}=\chi_{m, 1}+\chi_{n, 2}^{\prime}$, i.e., $\chi_{m}=\chi_{m, 1}$ independent on part 2 and $\chi_{n}^{\prime}=\chi_{n, 2}^{\prime}$ independent on part 1 .

\section{B. Distribution of delay times}

The considerations in the previous Sec. IV A also show that the statistical distribution of $\tau$ becomes independent of

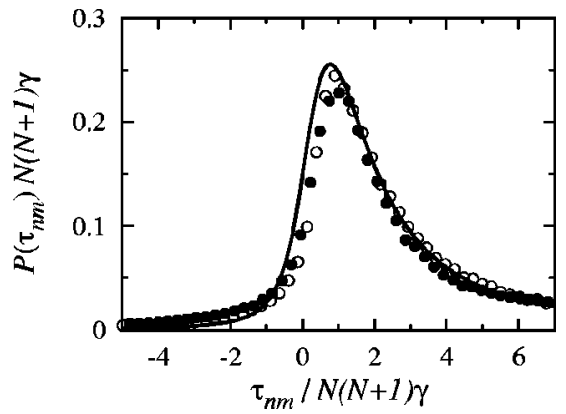

FIG. 3. Distribution of transmission delay time $\tau_{n m}$ for multichannel waveguides with $N=2$ (open dots) and $N=30$ (full dots). The analytic prediction from Eq. (29) (curve) with $Y=Z=\gamma N(N$ $+1) / 2$ is compared with the results of a numerical simulation of random scattering in a planar waveguide.

length ("stationary") for $L \gg \xi$, because the distribution $P\left(\chi_{m}\right)$ for length $L$ is identical to $P\left(\chi_{m, 1}\right)$ for length $L_{1}$ $<L$, and analogously for $\chi_{n}^{\prime}$.

The stationary distribution $P(\tau)$ is plotted in Fig. 3, for $N=2$ and $N=30$ propagating modes in the numerical simulations (corresponding to different widths $W$ of the waveguide). The distributions collapse onto a single curve when the delay times are rescaled by a factor $\gamma N(N+1)$. The distribution is however qualitatively different from the result for $N=1$. Most notably, a tail $\propto \tau_{12}^{-2}$ also extends into the region of negative delay times, while the delay times for $N=1$ are strictly positive.

An analytic treatment of the transmission delay-time problem for many channels is notoriously difficult. In the framework of one-dimensional scaling theory, the evolution of $\chi$ couples to all elements of $u$ and $d u / d \omega$, which makes a complete analytic solution impossible. Inspection of the complicated full set of evolution equations that appear in this approach, however, suggests the following approximation for the stationarity requirement of $P(\chi)$ :

$$
\left(-Y+\frac{\partial}{\partial \chi}\left(\chi^{2}+Z^{2}\right)\right) P(\chi)=0,
$$

with the solution

$$
P(\chi)=\frac{Y \exp [(Y / Z) \arctan (\chi / Z)]}{2\left(\chi^{2}+Z^{2}\right) \sinh (\pi Y / 2 Z)} .
$$

For $Y=\gamma$ and $Z=0$, the stationarity condition reduces to Eq. (20) for $N=1$. For $N>1$, the appearance of $Z$ can be traced back to the additional degrees of freedom in $u^{\dagger} d u / d \omega$, especially also to the real part of this matrix (the real part vanishes for $N=1$ ). This will be further discussed in the following two subsections IV C and IV D. The factor $Y / Z$ in the exponent of Eq. (29) determines the asymmetry of the distribution for positive and negative values of $\chi$.

The full set of evolution equations suggests that $Y \simeq Z$ $\simeq \gamma N(N+1) / 2$, up to numerical factors that cannot be derived without solving the original problem. This is also the order of magnitude of $\tau_{n m}$ at the border of diffusion and localization, see Eq. (8). In Fig. 3 we have plotted the distri- 
bution of $\tau=\chi+\chi^{\prime}$, which follows from Eq. (29) for $Y$ $=\gamma N(N+1) / 2$ and $Z=\gamma N(N+1) / 2$. The comparison with the numerical data shows that the numerical factors are close to unity.

\section{Relation to the reflection problem}

For $N=1$ we could relate the problem of transmission delay-times directly to the problem of reflection delay times. Now we discuss to which extent these two problems are linked for $N>1$.

Due to its symmetry, the scattering matrix can always be written as $S=U^{T} U$. In terms of the matrices of the polar decomposition, we can choose

$$
U=\left(\begin{array}{cc}
(\mathcal{T} / 2 p)^{1 / 2} u & (p / 2)^{1 / 2} v \\
-i(p / 2)^{1 / 2} u & i(\mathcal{T} / 2 p)^{1 / 2} v
\end{array}\right),
$$

with $p=1-\sqrt{1-\mathcal{T}}$. In the localized regime, $U$ can be approximated by

$$
U=\left(\begin{array}{cc}
u & (\sqrt{\mathcal{T}} / 2) v \\
-i(\sqrt{\mathcal{T}} / 2) u & i v
\end{array}\right) .
$$

The first index of the matrix $U$ is decorated by the transmission amplitudes, hence $U$ relates the scattering states to the transmission channels (each transmission channel is characterized by two vectors: a row of $u$ that connects it to the scattering states on the left and a row of $v$ that connects it to the scattering states on the right).

The Wigner-Smith time-delay matrix of the total scattering matrix is

$$
\begin{gathered}
Q=-i S^{\dagger} \frac{d S}{d \omega}=U^{\dagger} Q^{\prime} U, \\
Q^{\prime}=-i U^{*} \frac{d U^{T}}{d \omega}-i \frac{d U}{d \omega} U^{\dagger} .
\end{gathered}
$$

From the unitarity of $U$ is follows that $Q^{\prime}$ is real and symmetric, and hence diagonalized by an orthogonal matrix, which we write in block form

$$
O=\left(\begin{array}{ll}
o_{11} & o_{12} \\
o_{21} & o_{22}
\end{array}\right) .
$$

In this block form we denote the set of eigenvalues as $\operatorname{diag}\left(\tilde{\tau}, \tilde{\tau}^{\prime}\right)$. The matrix $O$ diagonalizes the Wigner-Smith matrix $Q$ on the basis of transmission channels, given by $U$, and hence relates the transmission channels to the eigenvectors of the Wigner-Smith matrix.

It is consistent to assume that $O$ is almost block diagonal, with off-diagonal elements $o_{12}, o_{21}$ of order $\sqrt{T_{1}}$. From $Q^{\prime}=O \operatorname{diag}\left(\tilde{\tau}, \tilde{\tau}^{\prime}\right) O^{T}$ we indeed obtain under this assumption the relations

$$
o_{11} \tilde{\tau} o_{11}^{T}=2 \operatorname{Im} u^{*} \frac{d u^{T}}{d \omega}
$$

$$
\begin{gathered}
o_{22} \tilde{\tau}^{\prime} o_{22}^{T}=2 \operatorname{Im} v^{*} \frac{d v^{T}}{d \omega} \\
o_{21} \tilde{\tau} o_{11}^{T}+o_{22} \tilde{\tau}^{\prime} o_{12}^{T}=\operatorname{Re}\left(\sqrt{\mathcal{T}} u^{*} \frac{d u^{T}}{d \omega}+\frac{d v^{*}}{d \omega} v^{T} \sqrt{\mathcal{T}}\right)
\end{gathered}
$$

Comparison with Eq. (11) shows that $o_{11}$ and $o_{22}$ diagonalize the Wigner-Smith matrices of the reflection problem (Sec. II F), however, in the special basis of transmission channels that is not fixed by reflection alone. The matrices $o_{12}$ and $o_{21}$ are related to frequency derivatives of $u$ and $v$ that do not feature in the reflection problem at all. Moreover, because they appear as off-diagonal elements of $O$, these matrices connect the coefficients of the transmission channels from one side of the waveguide to Wigner-Smith eigenvectors of reflection from the other side.

According to Eqs. (35) and (36), the eigenvalues of $Q$ can be approximated by the two sets $\tilde{\tau}, \tilde{\tau}^{\prime}$ of Wigner-Smith delay times of the reflection matrices. The transmission block of

$$
\frac{d S}{d \omega}=i U^{T} O \operatorname{diag}\left(\tilde{\tau}, \tilde{\tau}^{\prime}\right) O^{T} U
$$

corresponds to

$$
\begin{aligned}
\tau_{n m}= & \operatorname{Re} \frac{\left[v^{T}\left(\sqrt{\mathcal{T}} o_{11}+2 i o_{21}\right) \tilde{\tau} o_{11}^{T} u\right]_{n m}}{2\left(v^{T} \sqrt{\mathcal{T}} u\right)_{n m}} \\
& +\operatorname{Re} \frac{\left[v^{T} o_{22} \tilde{\tau}^{\prime}\left(o_{22}^{T} \sqrt{\mathcal{T}}+2 i o_{12}^{T}\right) u\right]_{n m}}{2\left(u^{T} \sqrt{\mathcal{T}} v\right)_{n m}} .
\end{aligned}
$$

Note that the separation (23) of the transmission delay time into two contributions that only depend on the incident or the detected mode is not evident from Eq. (39) [it follows, however, from Eq. (37)].

The main conclusion from Eq. (39) is that one cannot neglect the matrices $o_{12}, o_{21}$. That they appear here demonstrates that the reflection and transmission problem for $N$ $>1$ are not directly related. It is tempting to interpret the additional fluctuations from these matrices as the origin of the quantity $Z$ in Eq. (28).

In the next subsection we discuss an intensity-weighted combination of all transmission delay times that does not depend on $o_{12}$ and $o_{21}$.

\section{Weighted delay time and interpretation of long reflection delay times}

The matrix $O$, Eq. (34), carries the correlations of the transmission channels and the eigenvectors of the WignerSmith matrix ("delay-time channels"). A suitable object that captures the essence of these correlations can be formed with help of the intensity-weighted delay times 


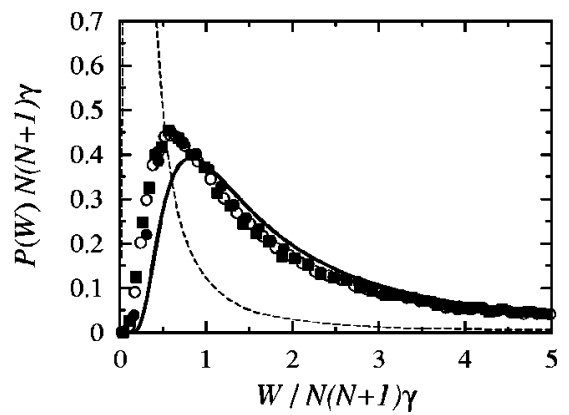

FIG. 4. Distribution of intensity-weighted combination $W$ of all transmission delay time for multichannel waveguides with $N=2$ (full dots), $N=5$ (open dots), and $N=10$ (squares), from the numerical simulation. The full curve is distribution $P\left(W_{\max }\right)$, Eq. (44), of the upper bound $W_{\max }$. The dashed curve is the result for $N$ $=10$ if the matrices $o_{11}$ and $o_{22}$ in Eq. (42) would be random orthogonal matrices.

$$
\begin{aligned}
W_{m n} & =\frac{\operatorname{Im}\left(d t_{n m} / d \omega\right) t_{n m}^{*}}{\operatorname{tr} t^{\dagger} t} \\
& =\operatorname{Im} \frac{u_{1 m}}{d \omega} u_{1 m}^{*}\left|v_{1 n}\right|^{2}+\operatorname{Im} \frac{v_{1 n}}{d \omega} v_{1 n}^{*}\left|u_{1 m}\right|^{2},
\end{aligned}
$$

where the last equality holds in the localized regime. The sum of all weighted delay times can be written as

$$
W=\operatorname{Im} \frac{\operatorname{tr}(d t / d \omega) t^{\dagger}}{\operatorname{tr} t^{\dagger} t}
$$

From Eq. (38) we find the representation

$$
\begin{aligned}
W & =\frac{\operatorname{tr} \mathcal{T}\left(o_{11} \tilde{\tau} o_{11}^{T}+o_{22} \tilde{\tau}^{\prime} o_{22}^{T}\right)}{2 \operatorname{tr} \mathcal{T}} \\
& =\frac{1}{2}\left[o_{11} \tilde{\tau} o_{11}^{T}+o_{22} \tilde{\tau}^{\prime} o_{22}^{T}\right]_{11},
\end{aligned}
$$

where the first diagonal element is picked out because the transmission eigenvalue $T_{1}$ is much larger than the other transmission eigenvalues. Hence $W$ indeed carries information of the correlations between the dominant transmission channel and the delay-time channels, which can be quantified by the overlaps

$$
\widetilde{o}_{i}=\left[o_{11}\right]_{1 i}, \quad \widetilde{o}_{i}^{\prime}=\left[o_{22}\right]_{1 i} .
$$

Note that $W$ does not involve the off-diagonal blocks $o_{12}$ and $o_{21}$ of $O$ that couple both ends of the waveguide, and that $W$ is manifestly positive.

The distribution $P(W)$ is plotted in Fig. 4 for some values of $N$ in units $\gamma N(N+1)$. These distributions are close to the distribution

$$
\begin{aligned}
P\left(W_{\max }\right)= & \frac{[N(N+1) \gamma]^{2}}{W_{\max }^{3}} \exp \left(-\frac{N(N+1) \gamma}{W_{\max }}\right) \\
& \times\left[K_{0}\left(\frac{N(N+1) \gamma}{W_{\max }}\right)+K_{1}\left(\frac{N(N+1) \gamma}{W_{\max }}\right)\right]
\end{aligned}
$$

of the mean

$$
W_{\max }=\frac{1}{2}\left(\tilde{\tau}_{1}+\tilde{\tau}_{1}^{\prime}\right)
$$

of the two largest delay times $\tilde{\tau}_{1}, \tilde{\tau}_{1}^{\prime}$, which follows from Eq. (13). Figure 4 also shows the distribution function if $o_{11}$ and $o_{22}$ would be random orthogonal matrices, which would result in much smaller values $W \simeq \gamma N$.

The quantity $W_{\max }$ is an upper bound of $W$. That the distributions of both quantities are very close and require a large overlap,

$$
\widetilde{o}_{1} \simeq \widetilde{o}_{1}^{\prime} \simeq 1
$$

of the dominant transmission channel with the channel with the largest delay time, hence, that both channels are strongly correlated. The correlation is strongest for large $W_{\max }$, because the tails of the two distributions coincide very well.

Upon reflection, the strong correlations of the dominant transmission channel and the channel with the largest delay time can be seen as one reason why the single-mode delay times $\tau_{n m}$ are of order $\gamma N(N+1)$, which corresponds to $Y$ $\simeq Z \simeq \gamma N(N+1) / 2$ in Eq. (28).

\section{CONCLUDING REMARKS}

In this paper we have investigated the statistical properties of the transmission delay time $\tau$ in the presence of wave localization. Most of the analysis relied on the separation of the delay time into two independent contributions, $\tau=\chi$ $+\chi^{\prime}$, with $\chi$ and $\chi^{\prime}$ given in Eq. (23). The properties of the delay time follow then from the distribution function of $\chi$ and $\chi^{\prime}$. This distribution does not depend on length in the localized regime. It is given as an exact analytic expression for $N=1$ in Eq. (21) and in approximate form for $N>1$ in Eq. (29).

We also have demonstrated in Sec. IV D that the dominant transmission channel is closely related with the channel associated to the largest Wigner-Smith delay time. Large reflection delay times can hence be interpreted as exploration of regions deep inside the waveguide, which are only accessible via the dominant transmission channel.

The separation rule (23) entails strict correlations among the delay times of a single realization, which are related by Eq. (24). These relations become invalid when absorption dominates over localization (then diffusion theory becomes applicable again). It would be interesting to investigate whether the departure from Eq. (24) qualifies as a practical tool that distinguishes these two distinct mechanisms of wave attenuation. 


\section{ACKNOWLEDGMENTS}

I thank C. W. J. Beenakker and K. van Bemmel for stimulating discussions. Part of this work was supported by the "Nederlandse organisatie voor Wetenschappelijk Onderzoek" (NWO) and by the "Stichting voor Fundamenteel Onderzoek der Materie" (FOM).

\section{APPENDIX: DISTRIBUTION FUNCTION OF $\chi$ FOR $N=1$}

The distribution $P(\chi)$ for $N=1$, Eq. (19), follows from the requirement of stationarity on its evolution equation (20).

In this Appendix we briefly sketch how the evolution equation is derived within one-dimensional scaling theory $[7,29,33,34]$, adapted to the dynamical problem along the lines of Refs. [23,35,36].

In this approach we study the evolution of $\chi(L+\delta L)$ $=\chi(L)+\delta \chi$ as the length of the waveguide is increased gradually, by adding a thin slice of length $\delta L$. Within an ensemble of random disorder, the evolution of the distribution function is then governed by a Fokker-Planck equation,

$$
\delta L \frac{\partial P}{\partial L}=\frac{\partial}{\partial \chi}\left(-\langle\delta \chi\rangle+\frac{1}{2} \frac{\partial}{\partial \chi}\left\langle\delta \chi^{2}\right\rangle\right) P(\chi) .
$$

In order to show that Eq. (A1) becomes Eq. (20), it remains to calculate the moments $\langle\delta \chi\rangle$ and $\left\langle\delta \chi^{2}\right\rangle$. The scattering matrix elements

$$
r_{1}=-r_{1}^{\prime *}=i B, \quad t_{1}=t_{1}^{\prime}=1+i A-(a+b) / 2,
$$

of the slice are given by a Gaussian real number $A$ with variance $\left\langle A^{2}\right\rangle=a$ and the complex number $B$ with $\left\langle|B|^{2}\right\rangle$ $=b$. From $\left\langle\left|r^{2}\right|\right\rangle=\delta L / 2 l$ we obtain the relation to the mean free path $l=\delta L / 2 b$. The derivative $d A / d \omega=\delta L / c$, as appropriate for the quasiballistic motion through the small segment.

Now we have to determine the elements $u$ and $d u / d \omega$ for the composed system of length $L+\delta L$. From the composition rule (25) and the reduced form $t=u v \sqrt{T}$, Eq. (9), we obtain in the localized regime the prescription

$$
\begin{aligned}
u(L+\delta L)= & u\left(1+i A+i \operatorname{Re} B u^{2}-a / 2-b / 2\right), \\
\frac{d u}{d \omega}(L+\delta L)= & \frac{d u}{d \omega}\left(1+i A+i \operatorname{Re} B u^{2}-a / 2-b / 2\right) \\
& +u\left(i \frac{\delta L}{c}+2 i \operatorname{Re} B u \frac{d u}{d \omega}\right),
\end{aligned}
$$

where we denoted for simplicity the initial value $u(L)=u$. The increment of $\delta \chi$ is then given by

$$
\delta \chi=\frac{\delta L}{c}+2 \operatorname{Re} B u \frac{d u}{d \omega},
$$

and the moments are

$$
\langle\delta \chi\rangle=\frac{\delta L}{c}, \quad\left\langle\delta \chi^{2}\right\rangle=2 b \chi^{2}=\frac{2 \chi^{2}}{\gamma} \frac{\delta L}{c} .
$$

[1] A. Ishimaru, Wave Propagation and Scattering in Random Media (Academic, New York, 1978).

[2] P. Sheng, Scattering and Localization of Classical Waves in Random Media (World Scientific, Singapore, 1990).

[3] R. Berkovits and S. Feng, Phys. Rep. 238, 135 (1994).

[4] S. John, Phys. Today 44(5), 32 (1991).

[5] P. W. Anderson, Phys. Rev. 109, 1492 (1958).

[6] B. Kramer and A. MacKinnon, Rep. Prog. Phys. 56, 1469 (1993).

[7] C. W. J. Beenakker, Rev. Mod. Phys. 69, 731 (1997).

[8] N. Garcia and A. Z. Genack, Phys. Rev. Lett. 66, 1850 (1991).

[9] A. Z. Genack and N. Garcia, Phys. Rev. Lett. 66, 2064 (1991).

[10] D. S. Wiersma, P. Bartolini, A. Lagendijk, and R. Righini, Nature (London) 390, 671 (1997).

[11] F. Scheffold, R. Lenke, R. Tweer, and G. Maret, Nature (London) 398, 206 (1999).

[12] A. A. Chabanov, M. Stoytchev, and A. Z. Genack, Nature (London) 404, 850 (2000).

[13] E. P. Wigner, Phys. Rev. 98, 145 (1955).

[14] F. T. Smith, Phys. Rev. 118, 349 (1960).

[15] Y. V. Fyodorov and H.-J. Sommers, J. Math. Phys. 38, 1918 (1997).

[16] A. Z. Genack, P. Sebbah, M. Stoytchev, and B. A. van Tiggelen, Phys. Rev. Lett. 82, 715 (1999).
[17] A. Lagendijk, J. Gómez Rivas, A. Imhof, F. J. P. Schuurmans, and R. Sprik, in Photonic Crystals and Light Localization in the 21st Century, Vol. 563 of NATO Science Series, edited by C. M. Soukoulis (Kluwer, Dordrecht, 2001).

[18] B. A. van Tiggelen, P. Sebbah, M. Stoytchev, and A. Z. Genack, Phys. Rev. E 59, 7166 (1999).

[19] A. M. Jayannavar, G. V. Vijayagovindan, and N. Kumar, Z. Phys. B: Condens. Matter 75, 77 (1989).

[20] J. Heinrichs, J. Phys.: Condens. Matter 2, 1559 (1990).

[21] A. Comtet and C. Texier, J. Phys. A 30, 8017 (1997).

[22] C. Texier and A. Comtet, Phys. Rev. Lett. 82, 4220 (1999).

[23] C. W. J. Beenakker and P. W. Brouwer, Physica E (Amsterdam) 9, 463 (2001).

[24] H. Schomerus, K. J. H. van Bemmel, and C. W. J. Beenakker, Europhys. Lett. 52, 518 (2000).

[25] H. Schomerus, K. J. H. van Bemmel, and C. W. J. Beenakker, Phys. Rev. E 63, 026605 (2001).

[26] C. W. J. Beenakker, e-print cond-mat/0009061v2.

[27] C. J. Bolton-Heaton, C. J. Lambert, V. I. Fal'ko, V. Progodin, and A. J. Epstein, Phys. Rev. B 60, 10569 (1999).

[28] A. D. Mirlin, Phys. Rep. 326, 259 (2000).

[29] P. A. Mello and A. D. Stone, Phys. Rev. B 44, 3559 (1991).

[30] A. Edelman, Linear Algebr. Appl. 159, 55 (1991).

[31] B. White, P. Sheng, Z. Q. Zhang, and G. Papanicolaou, Phys. Rev. Lett. 59, 1918 (1987). 
[32] M. Titov and C. W. J. Beenakker, Phys. Rev. Lett. 85, 3388 (2000).

[33] O. N. Dorokhov, Pis'ma Zh. Éksp. Teor. Fiz. 36, 259 (1982) [JETP Lett. 36, 318 (1982)].

[34] P. A. Mello, P. Pereyra, and N. Kumar, Ann. Phys. (N.Y.) 181,
290 (1988).

[35] C. W. J. Beenakker, J. C. J. Paasschens, and P. W. Brouwer, Phys. Rev. Lett. 76, 1368 (1996).

[36] P. W. Brouwer, Phys. Rev. B 57, 10526 (1998). 